九州大学学術情報リポジトリ

Kyushu University Institutional Repository

\title{
Relationships between genome constitution and esterase composition in Brassica
}

\section{Eguchi, Hiromi}

Biotron Institute, Kyushu University

Matsui, Tsuyoshi

Biotron Institute, Kyushu University

https://doi.org/10.5109/22791

出版情報: 九州大学大学院農学研究院紀要. 15 (3)，pp.331-344，1969-07. Kyushu University バージョン：

権利関係 : 
Journal of the Faculty of Agriculture, Kyushu University, Vol. 15, No. 3

July 30, 1969

\section{Relationships between genome constitution and esterase composition in Brassica}

Hiromi EGUCHI* and Tsuyoshi MATSUI*

\section{Introduction}

It is known that the change of gene can be studied through the differences in enzyme molecules synthesized in different genetic lines. In this point of view, one of the useful methods which will bring the information of phylogenetic importance in cultivated plants could be taken as comparative analysis of enzymes synthesized in different species and strains. Brassica species are regarded as distinct ones on the cytogenetical ground ; there exist 3 kinds of monogenomic group ( $a a$, bb, and cc) and digenomic group (aabb, bbcc, and aacc), respectively (Morinaga, 1928; Morinaga and Fukushima, 1933; Manton, 1933 ; Sikka, 1940 ; Fukushima, 1945 ; Mizushima, 1952). Those plants contain relatively large amount of esterases in the plumules and young seedlings. Genetic control of esterase formation has been analyzed in D rosophila melanogaster (Ogita, 1962; Wright, 1963) and other animals.

Present paper deals with analyses of relationships between genome constitution and esterase composition in several Brassica species, employing the zymogram (Hunter and Marker-t, 1957).

\section{Materials and methods}

Brassica species used have been maintained in pedgree cultures in the Horticultural Laboratory of Kyushu University, and were listed in Table 1. Material seedlings were sampled 3 days after germination. Fifty

\footnotetext{
A part of this study was presented at the spring congress of Japanese Society for Horticultural Science in 3964.

* Biotron Institute, Kyushu University, Fukuoka.
} 
Table 1. Material species and varieties in Brassica.

\begin{tabular}{|c|c|c|c|}
\hline Species & $\begin{array}{l}\text { Genome } \\
\text { constitution }\end{array}$ & $\begin{array}{l}\text { Chromosome } \\
\text { number (n) }\end{array}$ & $\begin{array}{l}\text { Horticultural } \\
\text { variety }\end{array}$ \\
\hline B. pekinensis Rupr. & an & 10 & Shimoyama-hakusai \\
\hline B. rapa $\mathrm{L}$. & $a a$ & 10 & Shin-hakata-kabu \\
\hline$B$. campestris $\mathrm{L}$. & $a a$ & 10 & Kanzaki-hanana \\
\hline B. chinensis $\mathrm{L}$. & $a a$ & 10 & Seppaku-taisai \\
\hline B. japonica Sieb. & $a a$ & 10 & $\begin{array}{l}\text { Okute-shiraguki- } \\
\text { chisuji-kyona }\end{array}$ \\
\hline B. trilocularis Hook & $a^{\prime} a^{\prime}$ & 10 & \\
\hline B. tournefortii Gouan & $T T$ & 10 & $\longrightarrow$ \\
\hline B. nigra Koch & $b b$ & 8 & California brown \\
\hline B. oleracea L. var. botrytis D. C. & $c c$ & 9 & Early snow ball \\
\hline B. oleracea L. var. capitata D. C. & $c c$ & 9 & Yoshin \\
\hline B. oleracea L. var. acephala D. C. & $c c$ & 9 & Kale \\
\hline B. juncea Hemsel & $a a b b$ & 18 & Miike-takana \\
\hline B. cernua Coss. & $a a b b$ & 18 & \\
\hline B. carinata Braun & $b b c c$ & 17 & Harron \\
\hline B. napus L. & aacc & 19 & Rape \\
\hline
\end{tabular}

gm of the cotyledonary seedlings was frozen at $-20^{\circ} \mathrm{C}$ and homogenized in $50 \mathrm{ml}$ of $M / 30$ phosphate buffer of $p H$ 7.0. The homogenate was ultracentrifuged at $145,000 \times \mathrm{g}$. The supernatant was lyophilized and stored for the use to obtain esterase zymogram.

Agar-gel electrophoresis was employed for preparing the esterasc zymogram. Each gel medium for the electrophoresis was prepared with 0.7 gm of agar, and $2.0 \mathrm{gm}$ of polyvinyl-pyrrolidonc (Ogita, 1962) in $100 \mathrm{ml}$ of $\mathrm{M} / 50$ phosphate buffer of $p H$ 7.0. Agar-gel plates were made $2 \mathrm{~mm}$ in thickness and supported by grass plate. The lyophilized extract was restored with deionized water, and a piece of filter paper $(1.5 \mathrm{~mm}$ $\times 12.0 \mathrm{~mm}$ ) was saturated with the extract and was placed on the agar-gel plate. The extract diffused from the filter paper into agar-gel. After 40 minutes, the filter paper was removed and the extract in agar -gel plate was exposed to the stabilized voltage of $20 \mathrm{~V} / \mathrm{cm}$ at $0^{\circ} \mathrm{C}--5^{\circ} \mathrm{C}$ for 120 minutes. After the electrophoretic separation, 1 per cent solution of $\boldsymbol{\beta}$-naphthyl acetate was sprayed on the surface of the agar-gel as the substrate of esterases. The agar-gel plate was incubated at $35^{\circ} \mathrm{C}$ for 30 minutes. The substrate, $\beta$-naphthyl acetate diffused into the agar-gel and was hydrolyzed by each esterase separated electrophoretically at individual location on an agar-gel plate. Naphthanil diazo blue B was used as the dye coupler. 


\section{Results and discussion}

Esterase patterns at different growth stages of B. rapa: Extracts originated from seeds, seedlings (at cotyledonary stage), and from leaves of $B$. rapa, respectively were used for zymogram as shown in Fig. 1. Five bands of esterases designated as $A 1 \sim A 5$ were found in the young seedlings and also in the leaves. Al band migrated slowly toward cathode, while other bands migrated towards anode. There were no differences in electrophoretic mobilities between the esterases of the young seedlings and those of the leaves. The intensity of the esterase bands of the young seedlings was in general, stronger than that of the leaves. On the other hand, Al, A2, A5 bands and an immobile band locating at the original point, were found in the seeds. The A2 band of the seeds was stronger, and the A5 band was weaker than those of the young seedlings and of the leaves. The pattern of seedling esterases was similar to that of leaf esterases, but was quite different from that of seed esterases. These facts suggest that the estcrase composition changes through the germination at quite fast tempo, then it become almost constant during the growing period of the seedlings until the growth stage of the 7 th leaves.

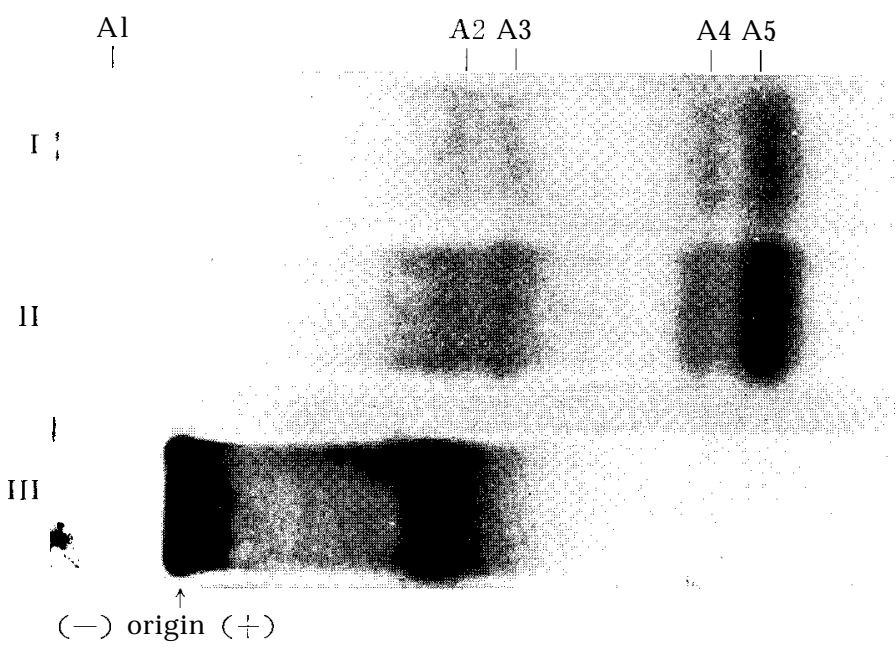

Fig. 1. Photograph of esterase zymogram showing the patterns of esterases occurred at various growing stages of B. rapa. (I) leaves set at the 4-5th nodes of the 5-7 leaf plants. (II) cotyledonary seedlings collected 3 days after germination. (III) seeds.

The behaviors of esterases under the treatment with $\beta$-mercaptoethanol:Betamercaptoethanol $(0.1 \mathrm{ml})$ was added to the extract $(10 \mathrm{ml})$ of $B$. rapa 
and of B. oleracea var. botrytis, and the treated extracts were used in zymograms. The treatment resulted in increasing the intensity of A2 and $\mathrm{A} 5$ bands in B. rapa, as well as $\mathrm{C} 2$ and $\mathrm{C} 6$ bands in B. oleracea. O $\mathrm{n}$ the other hand, A3 band in B. rapa and $\mathrm{C} 3$ band in $B$. oleracea were scarcely detectable in the treated extract. The $\beta$-mercaptoethanol had no effect on $\mathrm{Al}$ and $\mathrm{A} 4$ band of $\mathrm{B}$. rapa, and on $\mathrm{Cl}, \mathrm{C} 4, \mathrm{C} 5$ and $\mathrm{C} 7$ bands of B. oleracea. All the bands in the treated extracts were not different from those untreated in their electrophoretic mobilities as shown in Fig. 2. The esterases of A2, A5, C2 and C6 bands were activated by the treatment with $\beta$-mercaptoethanol. In most cases of the following experiments, $\boldsymbol{\beta}$-mercaptoethanol was added to the extract used for the zymograms.

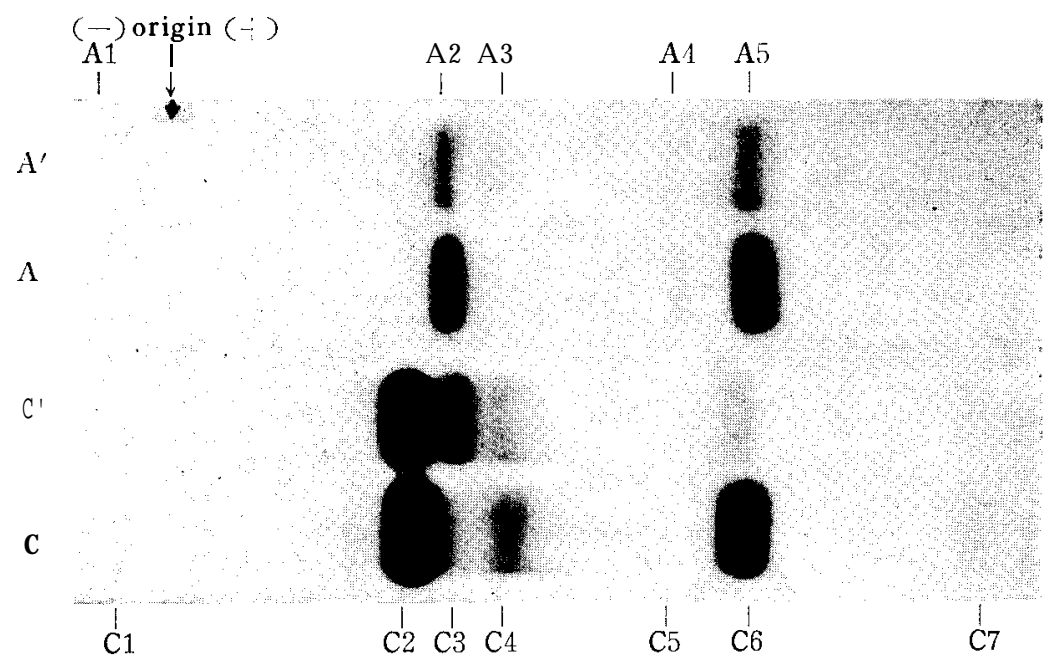

Fig. 2. Photograph of esterase zymogram showing comparison between the extract treated with $\beta$-mercaptoethanol and untreated extracts. ( $A^{\prime}$ ) untreated extract of B.rapa. (A) treated extract of B. rapa. $\left(\mathrm{C}^{\prime}\right)$ untreated extract of $\boldsymbol{B}$. oleracea var. botrytis. $\quad(\boldsymbol{C})$ treated extract of $\boldsymbol{B}$. oleracea var. botrytis.

Relationships between genome constitution and esterase composition : Fig. 3 shows the patterns of esterases in the seedlings of the $a$ genomic species and $\mathrm{c}$ genomic species. $\mathrm{Al}, \mathrm{A} 2, \mathrm{~A} 3, \mathrm{~A} 4$ and $\mathrm{A} 5$ bands were generally found in the $a$ genomic speces; the $\mathrm{Al}$ band which was very weak, migrated slowly towards cathode, and the other bands migrated towards anode. The A4 band in B. chinensis was weaker than that in B. rapa and B. pekinensis. There were no differences in electrophoretic mobilities of esterases among those 3 different species having aa genome, i. e., B. pekinensis, B. chenensis, and B. rapa. But 7 bands designated as $\mathrm{C} 1$, 
$\mathrm{C} 2, \mathrm{C} 3, \mathrm{C} 4, \mathrm{C} 5, \mathrm{C} 6$ and $\mathrm{C} 7$, were found in the c genomic species. The C3 band was not found in the extract treated with $\beta$-mercaptoethanol. The C5 and C7 bands were detected in var. botrytis only. All the bands of the c genomic species were different from those of the $a$ genomic species in their electrophoretic mobilities. Thus, inter-genomic differences between $a$ and $c$ genomic species could be observed in differential electrophoretic mobilities of their esterases. While the intra-genomic differences could be found in the differential intensity of certain bands. The differences among 3 species having the aa genome, i. e., B. pekinensis, B. chinensis and B. rapa, were taken to be on the same level as intra-specific differences among 3 varieties of $\boldsymbol{B}$. oleracea having cc genome, i. e., B. oleracea var. botrytis, var. capitata, and var. acephala. From this view-point, the degree of the differentiation existing among 3 different species having aa genome, are regarded

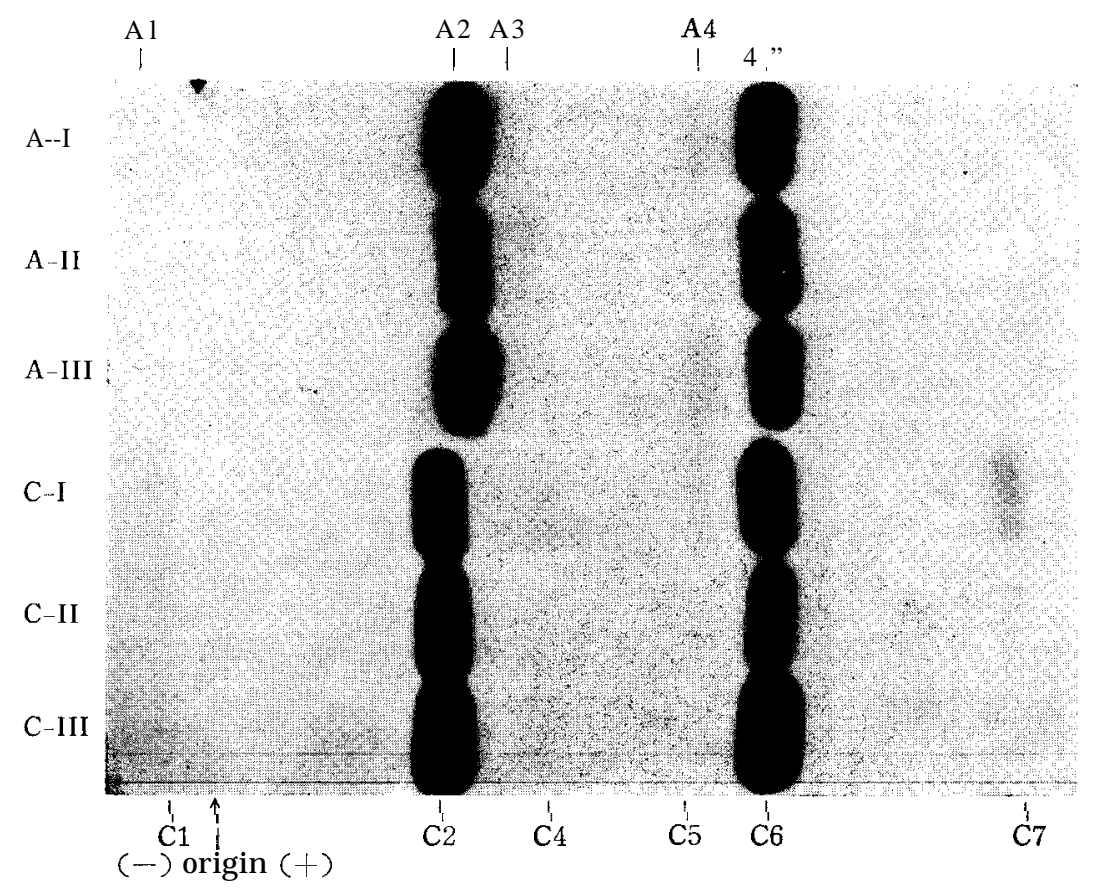

Fig. 3. Photograph of esterase zymograms derived from cotyledonary seedlings of Brassica, showing comparison between different monogenomic species (a a and cc genomes). (A-1) B. pekinensis (aagenome). (A-II) B. chinensis (aa genome). (A-III) B. rapa (aa ge nome). (C-l) B. oleracea var. botrytis (cc genome). (C-II) B. oleracea var. capitata (cc genome). (C-III) B. oleracea var. acephala (cc genome). 
as that being on a level with intra-specific differences.

Fig. 4 shows the relationship in esterase composition between an amphidiploid species, B.rapus (aacc genome) and the basic monogenomic species, B. pekinensis (au genome) and B. oleracea var. acephala (cc genome). An array of $\mathrm{Al}, \mathrm{C} 2, \mathrm{~A} 2, \mathrm{C} 4, \mathrm{C} 6$ and $\mathrm{A} 5$ bands were found in $\boldsymbol{B}$. napus. The C6 and A5 bands in B. napus were superimposed on each other, according to their close migration. The array of esterase bands in the amphidiploid species, B. napus, was found to be composed of the bands contained in the two basic monogenomic species.

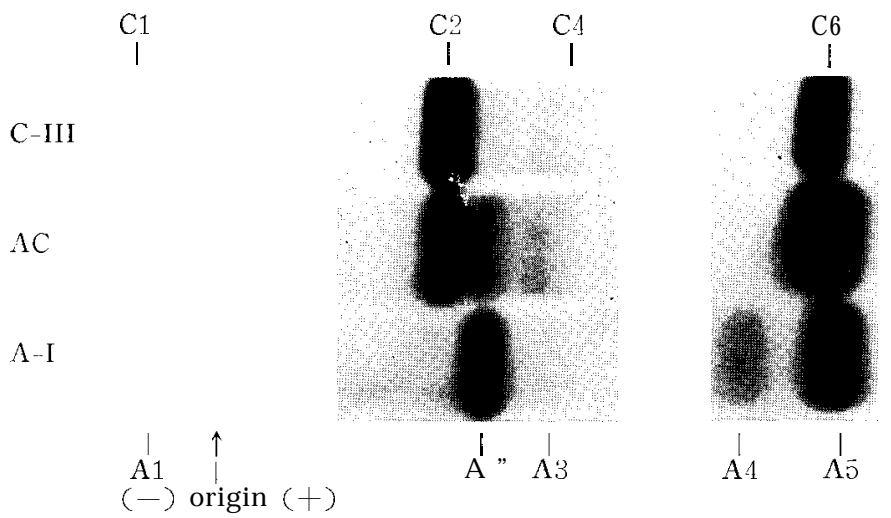

Fig. 1. Photograph of esterase zymogram derived from cotyledonary seedlings of Brassica, showing comparison between an amphidiploid species and its basic monogenomic species. (C-III) B. oleracea var. acephala (cc genome). (AC) B.napus (aacc genome). (A-I)B. pekinensis (au genome).

On the other hand, $\boldsymbol{b}$ genomic species, B. nigra ( $\boldsymbol{b} \boldsymbol{b}$ genomc) appeared to have 7 bands of esterases, designated as B1, B2, B3, B4, B5, B6 and B7, as shown in Fig. 5. The very weak B1 band migrated slowly towards cathode, while others towards anode. The B3 band which is closely adjacent to the position of the A3 band of a genomic species and the $\mathrm{C} 4$ band of $\mathrm{c}$ genomic forms, but the other 6 bands were distinctly different in their electrophoretic mobilities from those of other monogenomic species.

In an amphidiploid species, B. juncea (abb genome), Al, A2, B4 and A5 bands were found. The B4 band of B. juncea was stronger than that of B. nigra. The A2 and A5 bands of B. juncea were weaker than those of the a genomic forms. The A4 band was not clear in B. juncea, because the A4 band appeared to be superimposed on the strong B4 band. In another amphidiploid species having aabb genome, B. cernua, $\mathrm{Al}$, A2, X, A4 and A5 bands were found. The band designated as X could not be detected in either of the a genomic species or the $b$ genomic species 
as shown in Fig. 5. The A2 and A4 bands of $B$. cernua were weaker than those of the a genomic species

In B. carinata, an amphidiploid species having bbcc genome, $\mathrm{Cl}, \mathrm{C} 2, \mathrm{C} 4$, $\mathrm{X}, \mathrm{C} 6$ and $\mathrm{C} 7$ bands were found. This $\mathrm{Cl}$ band was very weak as quite like that of the $\mathrm{c}$ genomic species, and the $\mathrm{C} 2, \mathrm{C} 4, \mathrm{C} 6$ and $\mathrm{C} 7$ bands were definitely weaker than those of the $\mathrm{c}$ genomic species. It is strinkingly interesting that B. cernua (aabb genome) and B. carinata (bbcc genome) have the $\mathrm{X}$ band in common and have none of the bands which were present in B. nigra (bbb genome). The $\mathrm{X}$ band will be taken as the characteristic one of $\mathbf{b}$ genome. However, B. juncea (aabb genome) and B. nigra

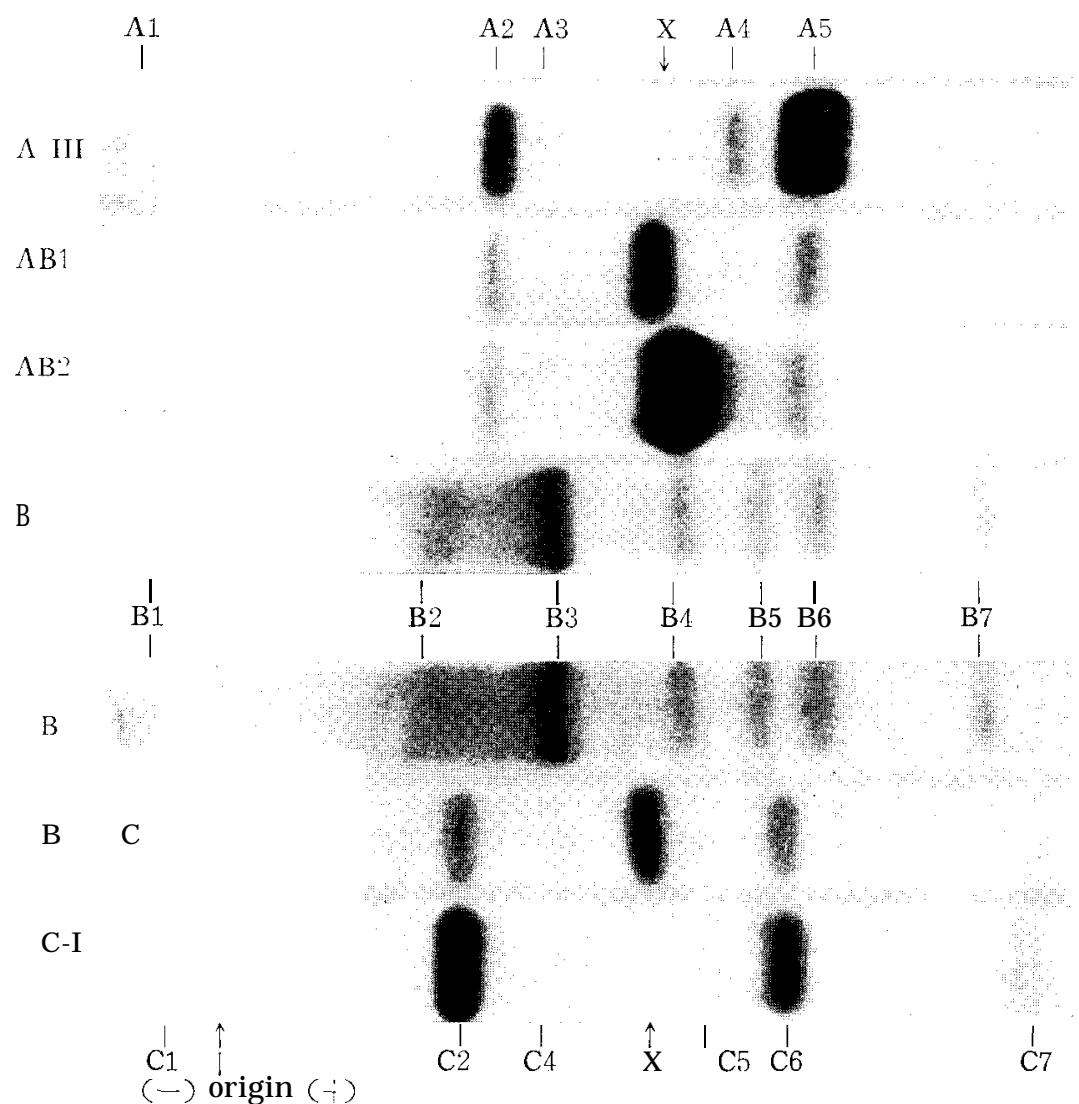

Fig. 5. Photograph of esterase zymograms derived from cotyledonary seedlings of Brassica, showing comparisons between different kinds of basic monogenomic species and the amphidiploid species. (A-III) B. rapa (aa genome). ( $\mathrm{AB} 1) \boldsymbol{B}$. cernua ( $a a b b$ genome). ( $\mathrm{AB} 2) \mathrm{B}$. juncea ( $a a b b$ genome). (B) $B$. nigra ( $b b$ genome). (BC) B. carinata (bbcc genome). (C-I) B. oleracea var. botrytis (cc genome). 
do not contain the $\mathrm{X}$ band. While, B. juncea and B. cernua have the A2 and A5 bands in common. It could not be considered that the same gene mutation responsible for the formation of the esterase had occurred in both of $\boldsymbol{B}$. carinata (bbcc gemone) and B. cernua (aabb genome) after spontaneous syntheses of these amphidiloid species and resulted in the production of the $\mathrm{X}$ band in both of those different amphidiploid species. It might be well conceivable that both of B. cernua and B. carinata had been spontaneously synthesyzed with the similar $\boldsymbol{b}$ genomic species having the $\mathrm{X}$ band, while B. juncea and B. cernua had been naturally synthesyzed independently with the different $\boldsymbol{b}$ genomic species and a similar $\boldsymbol{a}$ genomic species. From these considerations, it may be conceivable that the $\boldsymbol{b}$ genome of the amphidiploid species B. cernua and B. carinata, will be more or less different from the $\boldsymbol{b}$ genome of the present $\boldsymbol{b}$ genomic species, $\boldsymbol{B}$. nigra. Fukushima et al. (1968) performed serological analyses of leaf proteins in Brassica species and reported that the $\boldsymbol{b}$ genome of $\boldsymbol{B}$, carinata (bbcc genome) appears to be different from that of $\boldsymbol{B}$. nigra (bb genome) in their precipitin patterns. The results from the present experiments agree well with the serological evidence, and clearly point out that there exists certain genome-specific differences in the esterase patterns among the monogenomic species, having $\boldsymbol{a} a, b \boldsymbol{b}$, or cc genomes. It is also apparent that the esterase patterns of amphidiploid species are generally composed of the bands which are present in the basic monogenomic species except the $\mathrm{X}$ band. For example, B.napus (aacc genome) is quitely intermediate in its esterase pattern between the $a$ genomic species and the $\mathrm{c}$ genomic species The similar situation in the amphidiploid species was also observed in B. juncea (aabb genome), of which esterase pattern is composed of the bands of the $a$ genomic and the $\boldsymbol{b}$ genomic species On the other hand, B. cernua (aabb genome) has some of the bands of the a genomic spcies, but has none of the bands of $\boldsymbol{b}$ genomic species, $\boldsymbol{B}$. nigra. While $\boldsymbol{B}$. carinata has some of the bands of the c genomic species, but has none of $\boldsymbol{b}$ genomic species, B. nigra. As mentioned above, the $\mathrm{X}$ band is present in $\boldsymbol{B}$. carinata and $\boldsymbol{B}$. cernua. In view of these facts, B. cernua seems to be situated much closer to the $\boldsymbol{a}$ genomic species than to the $\boldsymbol{b}$ genomic species $\boldsymbol{B}$. nigra. But $\boldsymbol{B}$. carinata seems to be closer to the $c$ genomic species than to the $b$ genomic species. Furthermore, there exists a closer relationship between $\boldsymbol{B}$. cernua and $\boldsymbol{B}$. carinata than between either of these amphidiploid species and the $\boldsymbol{b}$ genomic species $\boldsymbol{B}$. nigra. The esterase bands detected in the present experiments were listed in Table 2.

In most cases, the intensities of the bands present in the amphidiploid species are different from those of the corresponding bands present in the basic species having monogenome in spite of their same electrophoretic mobilities. For example, the B4 band of B. juncea was 
Table 2-a. Esterase composition in cotyledonary seedlings of Brassica species.

\begin{tabular}{|c|c|c|}
\hline Species & $\begin{array}{c}\text { Genome } \\
\text { constitution }\end{array}$ & Esterase bands detected \\
\hline B. pekinensis & $a a$ & $\mathrm{Al}, \mathbf{A 2}, \mathrm{A} 3, \mathrm{~A} 4, \mathrm{~A} 5$ \\
\hline B. rapa & $a a$ & $\mathrm{Al}, \mathrm{A} 2, \mathrm{~A} 3, \mathrm{~A} 4, \mathrm{~A} 5$ \\
\hline B. chinensis & $a a$ & Al, A2, A3, A4, A5 \\
\hline B. nigra & bb & B1, B2, B3, B4, B5, B6, B 7 \\
\hline B. oleracea var. botrytis & cc & $\mathrm{Cl}, \mathrm{C} 2, \mathrm{C} 4, \mathrm{C} 5, \mathrm{C} 6, \mathrm{C} 7$ \\
\hline var. capitata & cc & $\mathrm{Cl}, \mathrm{C2}, \mathrm{C} 4, \quad \mathrm{C} 6$ \\
\hline var. acephala & $\mathrm{cc}$ & $\mathrm{Cl}, \mathrm{C} 2, \mathrm{C} 4$, \\
\hline B. juncea & aabb & Al, A2, B5, A5 \\
\hline B. cernua & aabb & $\mathrm{Al}, \mathbf{A} 2, \mathrm{X}, \mathrm{A} 4, \mathbf{A} \mathbf{5}$ \\
\hline B. carinata & bbcc & $\mathrm{Cl}, C 2, X, \mathrm{C} 6 . \mathrm{C} 7$ \\
\hline B. napus & aacc & $\mathrm{Al}, \mathrm{C} 2, \mathbf{A} 2, \mathrm{C} 4(=\mathrm{A} 3 ?), \mathrm{C} 6, \mathrm{~A} 5$ \\
\hline
\end{tabular}

N.B. Each of the extracts was treated with $\beta$-mercaptoethanol (1.0\%). Italic types show the bands with stronger intensity.

Bold-faces show the bands with the strongest intensity.

Table 2-b. Esterase composition in leaves obtained from the seedlings of various Brassica species having 10 pairs of chromosomes in somatic.

\begin{tabular}{lcc}
\hline \multicolumn{1}{c}{ Species } & Genome & Esterase bands detected \\
\hline B. pekinensis & $a a$ & $\mathrm{Al}, \mathrm{A} 2, \mathrm{~A} 3, \mathrm{~A} 4, \mathrm{~A} 5$ \\
B. rapa & $a a$ & $\mathrm{Al}, \mathrm{A} 2, \mathrm{~A} 3, \mathrm{~A} 4, \mathrm{~A} 5$ \\
B. campestris & $a a$ & $\mathrm{Al}, \mathrm{A} 2, \mathrm{~A} 3, \mathrm{~A} 4, \mathrm{~A} 5$ \\
B. chinensis & $a a$ & $\mathrm{Al}, \mathrm{A} 2, \mathrm{~A} 3, \mathrm{~A} 4, \mathrm{~A} 5$ \\
B. japonica & $a a$ & $\mathrm{Al}, \mathrm{A} 2, \mathrm{~A} 3, \mathrm{~A} 4, \mathrm{~A} 5$ \\
B. trilocularis & $a^{\prime} a^{\prime}$ & $\mathrm{Al}, \mathrm{A} 2, \mathrm{~A} 3, \mathrm{~A} 4, \mathrm{~A} 5$ \\
B. tournefortii & T T & $\mathrm{T} 1, \mathbf{T 2}$ \\
\hline
\end{tabular}

N. B. Italic types show the bands with stronger intensity.

Bold-faces show the bands with the strongest intensity.

stronger than that of B.nigra, while the A2 and A5 bands of B. juncea were weaker than those of B. rapa. Moreover, the $\mathrm{C} 2$ and $\mathrm{C} 6$ bands of B. carinata were weaker than those of B. oleracea as shown in Fig. 5. Wright (1963) reported a quantitative correlation between the intensity of an esterase band and the gene dosage responsible for its production in Drosophila melanogaster; each of the gene dose could produce approximately equal amount of enzyme activity, while a band produced by one dose of the gene, is about half as intense as that produced by two 
doses of the gene. In view of this evidence, it might be estimated that the dose of the gene responsible for the production of the esterase in an amphidiploid population would not be equal to that in the population of basic monogenomic species, composing such amphidiploid species ; for example, B. juncea population would possess a larger amount of genes responsible for the production of the esterase of B4 band than B. nigra population. These changes of the gene dosage might have occurred through evolutionary processes in nature and breeding in artificial force.

Such differences in the intensity of esterase bands were also observed among different species having aa genome. Fig. 6 illustrates comparative zymograms of leaf esterases present in different species having 10 pairs of chromosomes in somatic. The Al, A2, A3, A4 and A5 bands were generally found in the leaves of those species. These esterase patterns were similar to those in the seedlings of the a genomic species

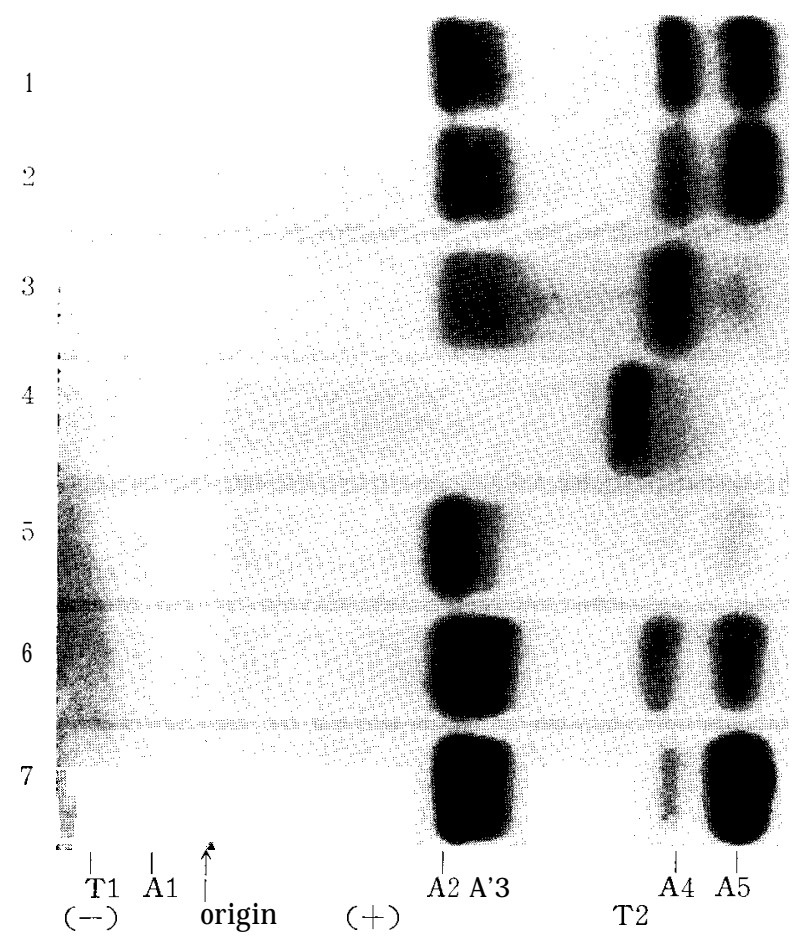

Fig. 6. Photograph of esterase zymograms derived from foliage leaves, showing the comparison among different Brassica species having 10 pairs of chromosomes in somatic. (Each extract used was not treated with $\beta$-mercaptoethanol.) (1) B. pekinensis (au genome). (2) B. rapa (aa genome). (3) B. campestris (a uenome). (4) B. tourneforfii (TT genome). (5) B. trilocularis (a'a' genome). (6) B. juponicu (au genome). (7) B. chinensis (au genome). 
shown in the above Figs. There were scarcely any appreciable differences in electrophoretic mobilities among different a genomic species, notwithstanding distinct differences among those species were found in intensities of the corresponding bands; the A4 band of B.chinensis was somewhat weaker, and the A5 band of B. campestris was much weaker than those of other a genomic species. Moreover, significant differences in the intensity were observed in the weaker bands, such as A3, A4 and A5 bands of B. trilocularis; especially the A4 band of B. trilocularis were so weak that it was scarcely detectable. These facts suggest that there would be significant differences in dose of genes responsible for the esterase production: A population of B. campestrissccms to possess small doses of the gene responsible for the production of A5 band esterase, furthermore, that of B. trilocularis seems to have smaller doses of genes reponsible for the production of A3, A4 and A5 band esterases. In view of these facts, it would be conceivable that B. trilocilaris seems to be more different from the other species having $a a$ genomes.

On the other hand, B. tournefortii showed a specific array of at least two bands, designated as T1, T2, and anindistinct band which is faster towards anode in its migration than the T2 band, not being discriminated according to its faint appearance. The T1 band showed remarkably weaker intensity and migrated slowly towards cathode and was somewhat faster in its migration than the $\mathrm{Al}$ band of the other species. The T2 band, which migrated towards anode, was slower in its migration than the A4 band of the other species. In view of this fact that the esterase pattern of B. tournefortii was different in electrophoretic mobilities from those of the other species having 10 pairs of chromosomes in somatic, it could be estimated that B. tournefortii would be different from the other species having $a a$ genome on a quite level with an inter-genomic difference.

Fukushima and Iwasa (1966) succeeded in discriminating thegenomes of B. trilocularis and B. tournefortii; the genome of B. trilocularis has been designated as $\mathbf{a}^{\prime}$, according to the evidence that the meiotic chromosome associations are shown as $(9-10) \mathrm{II} \dashv \cdot(2-0) \mathrm{I}$ in the $\mathrm{F}$, hybrid raised by the cross between B. trilocularis and B. campestris. That is, the genome of B. trilocularis is more or less different from the original a genome to some extent. While the genome of B. tournefortii has been designated as $T$, according to the evidence that B. tournefortii is highly cross incompatible with several Brassica species having $\mathbf{a}, \mathbf{b}$ or $\mathbf{c}$ genomes, and the meiotic chromosome associations are shown as (O-5) $I I+(20-10) I$ in the $\mathrm{F}$, hybrid raised by cross between $\mathbf{B}$, tournefortii and $\mathbf{B}$. campestris, that is, B. tournefortii is composed of a certain genome differing from the a genome and others. 
The results obtained through the present comparative analyses of leaf esterases of Brassica species having 10 pairs of chromosomes agree well with those cytogenetical evidences. That is, it could be estimated that B. tournefortii has completely different from the a genomic species in spite of their same numbers of chromosomes in somatic.

The comparative analyses of esterase compositions by means of zymogram were remarkably useful for the phylogenetic investigation of Brassica species, and could offer the following working hypotheses applicable for the phylogenetic studies of cultivated plants.

1) Inter-genomic difference : An array of esterase bands present in one species is different in electrophoretic mobilities of esterases.

2) Intra-genomic difference : Generally the esterase bands are different in intensities, and in some cases, a certain band is absent, though the other bands show the same electrophoretic mobilities.

3) Relationship in esterase composition between an amphidiploid species and their basic monogenomic species : The array of bands present in an amphidiploid form was mostly composed of some of the bands present in their basic monogenomic species. In some cases, the amphidiploid species does not contain the bands present in one of the basic species, but contains some of the bands present in the other one of the basic species and have the band which is not contained in either of the basic species.

The differences in esterase composition among various species of cultivated plants would show nothing but resuls of the change of several genes responsible for production of those esterases. However, the artificial selection for the esterase composition has not carried out; the differences in esterase composition among the species would be brought by the indirect result of differentiations subsequent to natural selection over the long period of evolutionary history and artificial selection for the other characters. Consequently, the variation in esterase composition could reflect the fundamental difference in gene pool of the population,

\section{Summary}

Relationships between genome constitution and esterase composition in Brassica species, were analyzed by means of the zymogram, and the following results were obtained:

1) The differences among the a genomic species (Brassicarapa and others), the $b$ genomic species (B. nigra), and the c genomic species (B. olerasea), could be observed in electrophoretic mobilities of their esterases. 
2) Intra-genomic differences could be observed in differential intensities of some bands, and in some cases, observed in existence of some specific bands.

3) The array of the csterase bands in amphidiploid species of Brassica is composed of some of the bands present in the basic species having monogenome. Some of the amphidiploid species contain the bands present in one of their basic species, but have none of the bands present in the other one of their basic species, and further, they have a band which is not present in either one of the basic species ; $B$. juncea (aabb genome) has some of the bands present in the a genomic species and the b genomic species, while $\mathbf{B}$. cernua (aabb genome) has some bands present in the a genomic species, but has none of the bands present in the $\mathbf{b}$ genomicspecies, and further, has one band designated as $\mathrm{X}$, which is not contained in both of the basic species. Similar situation could be observed in B. carinata (bbcc genome), which has some of the bands present in the $\mathrm{c}$ genomic species and has the $\mathrm{X}$ band, but has none of the bands present in the $\mathbf{b}$ genomic species. These findings suggest that the b genome composing B. cernua (aabb genome) and B. carinata (bbcc genome) will be somewhat different from that of $\mathbf{B}$. juncea (aabb genome) and B. nigra (bb genome).

4) In comparing the Brassica species having 10 pairs of chromosomes in somatic, it became apparent that B. trilocularis is distinctly different from the other species having aa genome in the intensities of some of the esterase bands, and B. tournefortii is much more different from the other species in electrophoretic mobilities of all the esterase bands. These findings agree well with the cytogenetic evidences proved by Fukushima and Iwasa (1966) who have assigned $\mathbf{T}$ genome to $\mathbf{B}$. tournefortii and a' genome to B. trilocularis.

\section{Acknowledgment}

The authors wish to express their sincerest appreciation to Dr. E. Fukushima, Emer. Professor of Kyushu University, for his valuable guidance in the course of this work The authors are very happy to express their gratitude to Emer. Professor H. Itoh for his valuable advices.

\section{Literature cited}

1) Fukushima, E., 1945 Cytogenetic studies on Brassica and Raphanus. I, Studies on the intergeneric $F_{1}$ hybrids between Brassica and Raphanus. Jour. Dept. Agric. Kyushu Imp. Univ. 7: 281.

2) Fukushima, E., and S. Iwasa, 1966 Genome-analytic studies on Brassica tri- 
locularis and B. tournefortii. Jour. Fac. Agric. Kyushu Univ. 13(4): 743.

3) Fukushima, E., T. Matsui, and II. Eguchi, 1968 Phylogenetic studies on Brassica species by means of serological method. Jour. Fac. Agric. Kyushu Univ. 14 (3): 341.

4) Haga, T., 1938 On the genomes in the genus Brassica. (A collective review in Japanese). Jap. Jour. Genet. 14: 74.

5) Hunter, R.L., and C. L. Markert, 1957 Histochemical demonstration of enzymes separated by zone electrophoresis in starch gels. Science, 125: 1294.

6) Manton, I., 1932 Introduction to the cytology of the Crucifereae. Ann. Bot. 46: 509.

7) Mizushima, U., 1944 Haploid parthenogenesis in Brassica and Sinapis. Agric. and Hort. 19: 743 (in Japanese).

8) Morinaga, T., 1928 Preliminary note on interspecific hybridization in Brassica. Proc. Imp. Acad. Jap. 4: 620.

9) Morinaga, T., and E. Fukushima, 1933 Karyological studies on a spontaneous haploid mutant of B. Napella. Cytologia 4: 457.

10) Ogita, Z., 1962 Genetico-biochemical analysis on the enzyme-activities in the house fly by agar gel electrophoresis. Jap. Jour. Genet. 37: 518.

11) Sikka, S. M., 1940 Cytogenetics of Brassica hybrids and species. Jour. Genet. 40: 441.

12) Wright, T. R. F., 1963 The genetics of an esterase in D rosophila melanogaster. Genetics $48: 787$. 